\title{
Studies on the intrinsic nervous system of the wild rodent Calomys callosus digestive tract. II. The submucous plexus
}

N.B. Souza,

E.A. Liberti and R.R. De-Souza
Departamento de Anatomia, Instituto de Ciências Biomédicas, Universidade de São Paulo, São Paulo, SP, Brasil
Correspondence

E.A. Liberti

Departamento de Anatomia

$$
\text { ICB, USP }
$$

Av. Prof. Lineu Prestes, 2415

05508-900 São Paulo, SP

Brasil

Fax: 55 (011) 813-0845

Publication supported by FAPESP.

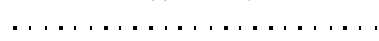

Received August 19, 1997

Accepted February 6, 1998

\section{Abstract}

The submucous plexus of the normal small and large intestine of Calomys callosus was studied by NADH and AChE histochemical techniques and by transmission and scanning electron microscopy. The plexus contains $(\bar{X} \pm \mathrm{SD}) 7,488 \pm 293$ neurons $/ \mathrm{cm}^{2}$ in the duodenum, 5,611 \pm 836 in the jejunum, 2,741 \pm 360 in the ileum, $3,067 \pm 179$ in the cecum, and 3,817 \pm 256 in the proximal colon. No ganglia or nerve cell bodies were seen in the esophagus, stomach, distal colon or rectum. The neurons are pear-shaped with a round or oval nucleus and the neuronal cell profile areas were larger in the large intestine than in the small intestine. Most of the neurons display intense AChE activity in the cytoplasm. AChE-positive nerve fibers are present in a primary meshwork of large nerve bundles and in a secondary meshwork of finer nerve bundles. At the ultrastructural level, the ganglia are irregular in shape and covered with fibroblastlike cells. The nucleoplasm of the neurons is finely granular with a few condensations of chromatin attached to the nuclear envelope. In the neuropil numerous varicosities filled with vesicles of different size and electron densities are seen. The pre- and post-synaptic membrane thickenings are asymmetric. Characteristic glial cells with oval nuclei and few organelles are numerous. These data provide a detailed description of this submucosal meshwork.

\section{Introduction}

Studies using both light (1-3) and scanning electron microscopy $(4,5)$ methods have demonstrated that the submucous plexus consists of a meshwork of connecting nerve strands which extend in various directions, which present ganglia at their junctions. The fine structure of this plexus was studied in the gut of animals such as fish $(6,7)$ and guinea-pigs (8).
Key words

- Submucous plexus

- Wild mouse

- Morphometry

- Histochemistry

- Ultrastructure

- Calomys callosus 
(9), particularly the submucous plexus.

In a previous study, we examined the morphology of the myenteric plexus of the digestive tract of Calomys callosus (10) in view of the fact that the different effects of chagasic infection on the enteric plexuses of different species may be due to the structure of the plexuses.

In another preliminary study, the effects of the Trypanosoma cruzi infection on quantitative and morphologic aspects of the neurons of the enteric plexus were compared between Swiss mice and Calomys callosus and the neuronal loss in Calomys callosus was found to be less than in mice (11).

In the present study, we describe the normal histomorphometry, using the NADH and $\mathrm{AChE}$ methods, and the fine structure of the submucous (Meissner) plexus of the Calomys callosus digestive tract. The results may contribute to future investigations of the same animal in many types of experiments, such as aging and infection with Trypanosoma cruzi.

\section{Material and Methods}

Histochemical dehydrogenase reaction

Five male adult Calomys callosus specimens weighing 30 to $35 \mathrm{~g}$ were used. The animals were sacrificed with an overdose of ether and the following regions of the gut were removed and washed in Krebs solution (12): approximately 4-cm lengths of the duodenum, mid-jejunum, ileum $(4 \mathrm{~cm}$ above the ileocecal junction), colon, and the entire cecum and rectum.

Each piece was then ligated with cotton thread at each end and gently distended with Krebs solution introduced at one end with a syringe. When sufficient distention had been achieved, the syringe needle was withdrawn and the ligature at that end simultaneously tightened.

The segments where placed under a dissecting microscope and the muscle layers were peeled away with tweezers from large areas, thus exposing the outermost aspect of the submucosa (12).

After being left for 15-30 min in Krebs solution at room temperature, the segments were transferred to a permeabilizing agent (a $0.3 \%$ solution of Triton- $\mathrm{X}$ in Krebs solution for 15-90 s) and submitted to 3 changes of Krebs solution for about $10 \mathrm{~min}$ and then incubated for $30-90 \mathrm{~min}$ at $20^{\circ} \mathrm{C}$ in $20 \mathrm{ml}$ of incubation medium containing nitro blue tetrazolium (Sigma Chemical Co., St. Louis, $\mathrm{MO}), 0.5 \mathrm{mg} / \mathrm{ml}$ in distilled water (25 parts), $0.1 \mathrm{M}$ sodium phosphate buffer, $\mathrm{pH} 7.3$ (25 parts), distilled water (50 parts) and B-nicotinamide adenine dinucleotide (reduced form), $0.5 \mathrm{mg} / \mathrm{ml}$.

The development of the reaction was monitored under a dissecting microscope and was stopped by immersion of the pieces in buffered $10 \%$ formalin, in which they were fixed for a minimum of $24 \mathrm{~h}$.

Laminar preparations of the pieces were then made as follows: three circular fragments $\left(12.56 \mathrm{~mm}^{2}\right)$ were obtained for each portion of the digestive tract; the submucosa of each fragment was freed of any remaining bundles of musculature and the mucosa scraped away. After several washes in distilled water, the submucosa was picked up on slides and mounted in glycerol, and all slides were sealed with Entellan (Merck).

Morphometry

The number of ganglion neurons and the profile of nerve cells were obtained by examining the whole-mount preparations under a binocular microscope at 400X magnification. All ganglia and neurons present in circular fragments were counted. No ganglia or nerve cell bodies were seen in the esophagus, stomach, distal colon or rectum preparations.

The profiles of 300 nerve cell perikarya for each segment of the digestive tract were outlined on drawing paper using a camera 
lucida attached to the microscope. The areas of these nerve cell bodies were calculated using a digitizing pad. Data were analyzed statistically by the Kruskal-Wallis nonparametric test (13) since the distribution of neurons was similar in the four portions studied, i.e., they did not present a normal distribution.

Demonstration of acetylcholinesterase

For the histochemical demonstration of acetylcholinesterase the direct coloring copper ferrocyanide method (14) was used in fresh tissues from five animals prepared as whole-mount stretch preparations.

Transmission electron microscopy

Three adult Calomys callosus specimens were used for transmission electron microscopy. Fixation was performed by intravascular perfusion of $4 \%$ glutaraldehyde in $0.1 \mathrm{M}$ phosphate buffer, $\mathrm{pH} \mathrm{7.2,} \mathrm{for} 10 \mathrm{~min}$. The abdomen was opened and small tissue pieces were dissected out from the distal ileum and proximal colon. The specimens were postfixed in phosphate-buffered $(0.1 \mathrm{M}) 1 \% \mathrm{OsO}_{4}$ solution for $2 \mathrm{~h}$, dehydrated with ethanol and embedded in Araldite. Semithin sections (1$\mu \mathrm{m}$ thick) were stained with $1 \%$ alcoholic toluidine blue and examined under the light microscope. Thin sections were cut with diamond knives on an ultramicrotome and stained with lead citrate and uranyl acetate. Electron micrographs were taken with a Philips EM 301 apparatus.

Scanning electron microscopy

Five adult Calomys callosus specimens were used. The fixation was performed as described for transmission electron microscopy and both the small and large intestines were removed.

Two centimeter wide segments were obtained from the middle part of each region opened to remove the mucosa and the extracellular material (15). The segments were then washed in phosphate buffer, dehydrated in an increasing alcohol series, and dried to the critical point with liquid $\mathrm{CO}_{2}$ in a Balzers CPD-010 apparatus, gold coated in a Balzers SCS-014 ion sputterer and examined with a Cambridge scanning electron microscope.

\section{Results}

Morphology of the submucous plexus

The submucous plexus studied by the NADH diaphorase method showed several ganglia containing variable numbers of neurons readily recognizable in all parts of the small intestine and in the cecum and proximal colon. The staining technique employed resulted in sharply delimited perikarya and clear visualization of the neural nuclei (Figure 1A). In all portions of the digestive tract, a single nerve cell body or a doublet was occasionally found (Figure 1B).

When using the AChE histochemical technique it was observed that the pattern of the plexus varied in different portions of the gut in which it was present (Figure 1D,F). The ganglia were connected with nerve bundles in a highly irregular arrangement and the plexus consisted of a primary meshwork of large bundles within which a secondary meshwork of finer fibers was also seen (Figure $1 \mathrm{D}, \mathrm{G})$. Most neurons stained intensely for AChE while a few stained only moderately (about $85 \%$ of the neurons showed a positive reaction for $\mathrm{AChE}$ ) (Figure 1C,G).

In the duodenum, jejunum and ileum the nerve cells were closely packed, whereas in the cecum and colon they were more widely spaced. The neurons were circular in profile, or slightly elongated, in the latter case without a preferential orientation with respect to the length and circumference of the gut (Figure $1 \mathrm{~A}, \mathrm{C})$.

Scanning electron microscopy showed 
that the submucous ganglia were small structures, irregular in shape and widely spaced. Their connecting nerve strands ran at several angles to the circular muscle, producing a network of roughly triangular, quadrangular or polygonal meshes (Figure 2A). Nerve fibers of the submucosal plexus passed through the circular muscle layer (Figure 2B). Ganglia and connecting strands were extensively covered with fibroblast-like cells. These cells were plate-like, with short processes which contacted and interlocked with each other (Figure 2C). Below the covering of periganglionic fibroblasts, the surface of the ganglion was covered by a basal lamina, which gave it a smooth appearance (Figure 2C,D).
Number of neurons

There were considerable variations in the number of neurons per unit surface area among the organs. The average number of neurons was 7,488 \pm 293 in the duodenum, $5,611 \pm 836$ in the jejunum, $2,741 \pm 360$ in the ileum, 3,067 \pm 179 in the cecum and $3,817 \pm 256$ neurons $/ \mathrm{cm}^{2}$ in the colum $(\overline{\mathrm{X}} \pm$ $\mathrm{SD})$.

Size of neurons

The average neuronal cell size was $123 \pm$ $86 \mu \mathrm{m}^{2}$ in the duodenum, $156 \pm 73 \mu \mathrm{m}^{2}$ in the jejunum, $166 \pm 94 \mu \mathrm{m}^{2}$ in the ileum, 318
Figure 1 - Whole-mount preparations of the submucous plexus of Calomys callosus. Ganglion (A) and isolated large neuron (B) in the small intestine; $C$, circular (arrow) or elongated (arrowhead) neurons in a large ganglion of the large intestine; $D-G$, primary meshwork of large nerve bundles (arrows) with a secondary meshwork of finer fibers (arrowheads) connecting the ganglia of the submucous plexus $(D, E$, small intestine; $F, G$, large intestine). (A,B - NADH, 170X; C-G - AChE). Scale bars: A, B, C, E, G, 100 $\mu \mathrm{m} ; \mathrm{D}, \mathrm{F}, 200 \mu \mathrm{m}$.

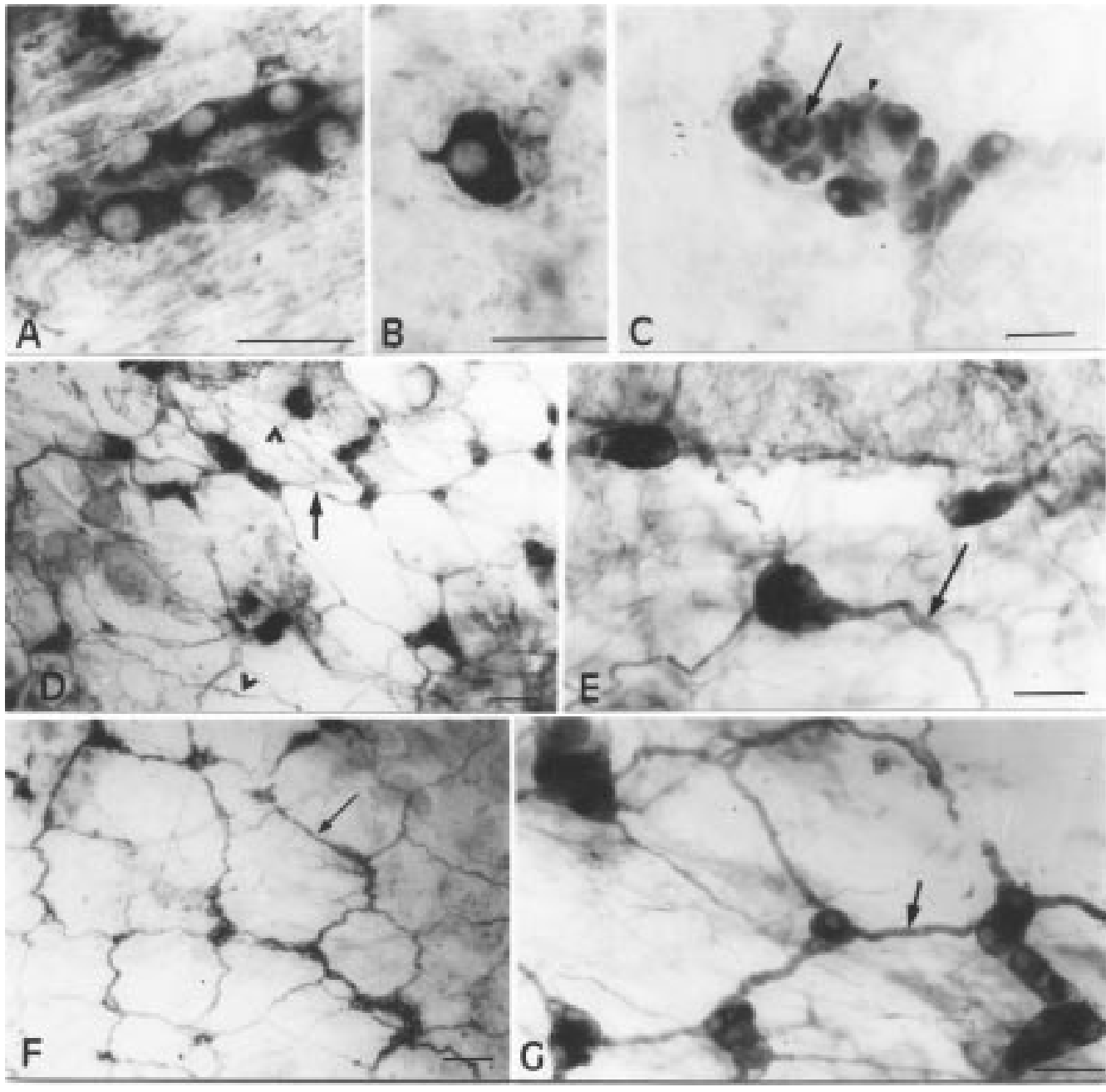


$\pm 191 \mu \mathrm{m}^{2}$ in the cecum, and $256 \pm 134 \mu \mathrm{m}^{2}$ in the colon. In the small intestine, the majority of neurons had neuronal cell profile areas between 50 and $150 \mu \mathrm{m}^{2}$; in the cecum and colon, the majority of neurons had neuronal cell profile areas between 100 and $300 \mu \mathrm{m}^{2}$.

\section{Ultrastructure of ganglia}

Transmission electron microscopy showed that the ganglion had a smooth surface and was surrounded by a continuous basal lamina (Figure 3B). Collagen fibers, fibroblasts, blood vessels, and interstitial spaces surrounded the ganglion near the basal lamina, but they did not penetrate inside the ganglion (Figure 3B,C). The presence of neurons and glial cells in close proximity to several axons and their varicosities were observed in each ganglion (Figure 3C).

The cell bodies of the neurons were pearshaped and had a large, round or oval nucleus (Figure 3A). The nucleoplasm was finely granular with a few condensations of chro- matin attached to the nuclear envelope. Ribosomes were the most common feature of the cytoplasm, arranged in rosettes. The cytoplasm also contained aggregations of free ribosomes, the Golgi apparatus (Figure 3C) and small round and oval mitochondria.

For the most part, the cell bodies of the neurons and axons were embedded in the infoldings of the glial cell cytoplasm. The axonal profiles contained prominent microtubules, mitochondria, and different types of vesicles (Figure 3C,D). The majority of the neurons had a part of their plasma membrane directly contacting the basal lamina surrounding the entire ganglion.

Glial cells were numerous in the ganglia and their bodies were generally smaller than those of neurons. In contrast to nerve cells, perikarya of glial cells were poorly equipped with cell organelles. The nuclei were oval, with deep indentations and large patches of dense material attached to the nuclear envelope. Processes of glial cells branched and spread among the nerve processes and neu-
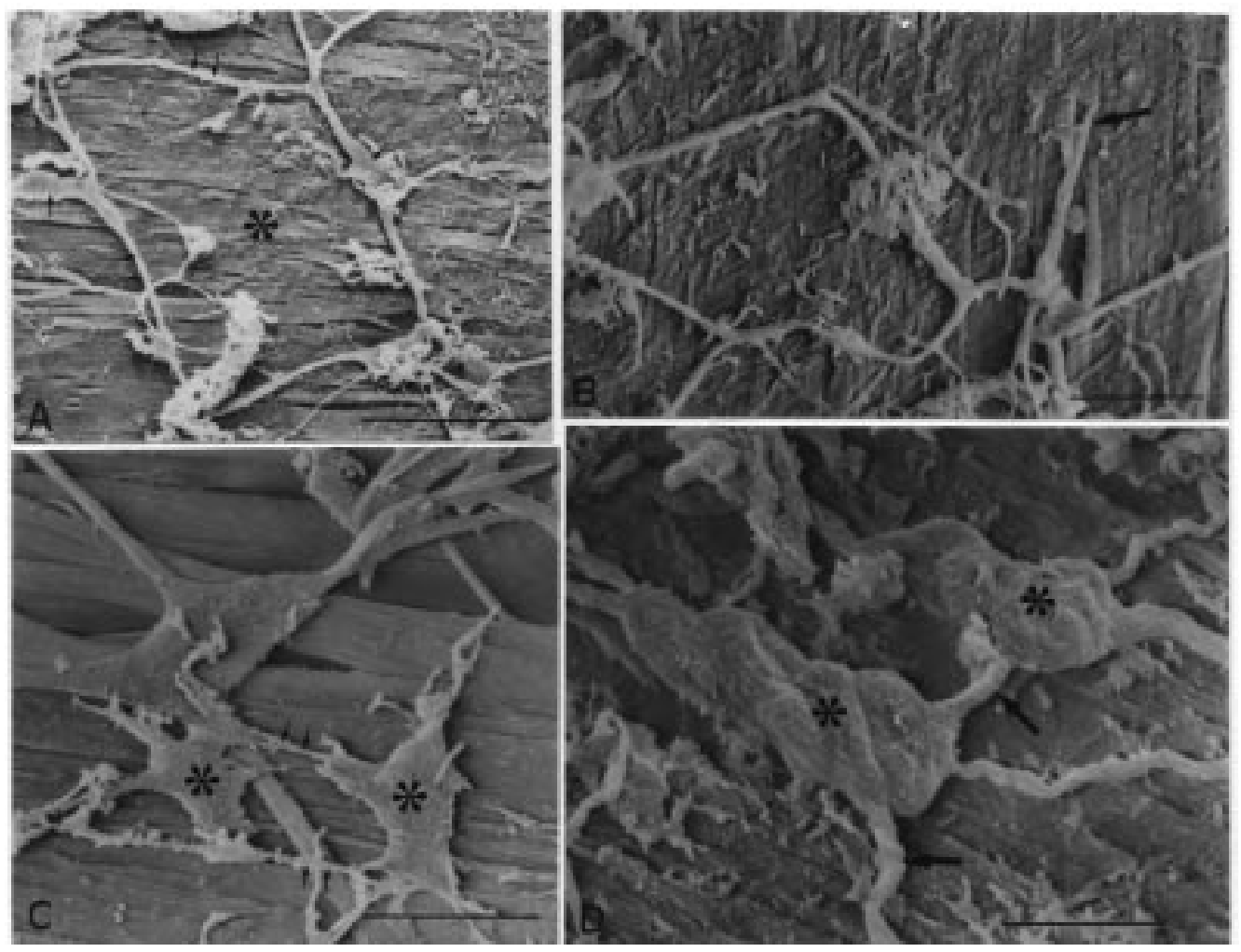

Figure 2 - Scanning electron microscopy of the submucous plexus of Calomys callosus. A, Ganglia and connecting nerve strands (arrows) close to the circular muscle layer of the intestine (*). $B, A$ nerve strand penetrating among the muscular fibers of the circular muscle layer (arrow). C, Fibroblast-like cells (*) with their characteristic short processes (arrows). D, Two ganglia (*) and connecting nerve strands (arrows). In $C$ and $D$ note the basal lamina which gives the ganglia a smooth appearance. Scale bars: A, $50 \mu \mathrm{m} ; \mathrm{B}, 20 \mu \mathrm{m}$; C,D, $10 \mu \mathrm{m}$. 
ron perikarya, some of them reaching the surface of the ganglia.

Inside the submucous ganglia, the axoplasm of the varicosities was filled with vesicles of different sizes and electrondensities (Figure 3C,D). Three types of vesicles were identified: small electronlucent vesicles, medium-sized granular vesicles, and large granular vesicles. The granular vesicles contained material of light electrondensity located centrally within their cores. This material was separated from the investing membrane by a narrow electronlucent zone (Figure 3D).

Most vesiculated nerve processes showed the membrane differentiation of typical synaptic junctions. The pre- and post-synaptic membrane thickenings were asymmetric, the post-synaptic membrane being more prominent. Synapses on perikarya and dendrites were formed by nerve processes with agranu- lar vesicles and with granular vesicles (Figure 3D).

\section{Discussion}

Although the functions of the submucous plexus are undoubtedly numerous, heterogeneous, and different among organs (2), the present observations regarding the morphology, histochemistry and fine structure of the submucous plexus of the digestive tract of Calomys callosus agree with the results obtained for other rodent species. The pattern of its submucosal plexus is similar to that of mice. Ganglia are normally located in the nodes of the plexus and a large number of thin connecting bundles emanate from each of them. As with the submucous plexus of mice, the meshes of the plexus are short and the ganglia are small $(1,16,17)$.
Figure 3 - Electron micrographs of the submucous plexus of Calomys callosus. A, The ganglion displays a pear-shaped nerve cell with a round nucleus ( $\mathrm{n}$ ) and a nucleolus. Around the ganglion are collagen fibrils (c) and processes of interstitial cells (i). B, Section through the neuropil (n) and an interstitial cell (i). In the neuropil, several glial and neural processes are visible. Collagen and a basal lamina (arrows) surround the ganglion. $C$, Section through the neuropil. A nerve ending with small agranular vesicle (a) synapses on a dendrite (d); in the latter, neurofilaments are visible. Endings containing mainly granular vesicles (g) are also present. The cytoplasm of a nerve cell shows the Golgi apparatus (arrows). D, A dendrite receives a synapse (arrow) from a nerve ending. The latter contains small clear vesicles and several large granular vesicles. Scale bars: A, $5 \mu \mathrm{m} ; \mathrm{B}, \mathrm{C}, 1$ $\mu \mathrm{m} ; \mathrm{D}, 0.5 \mu \mathrm{m}$.

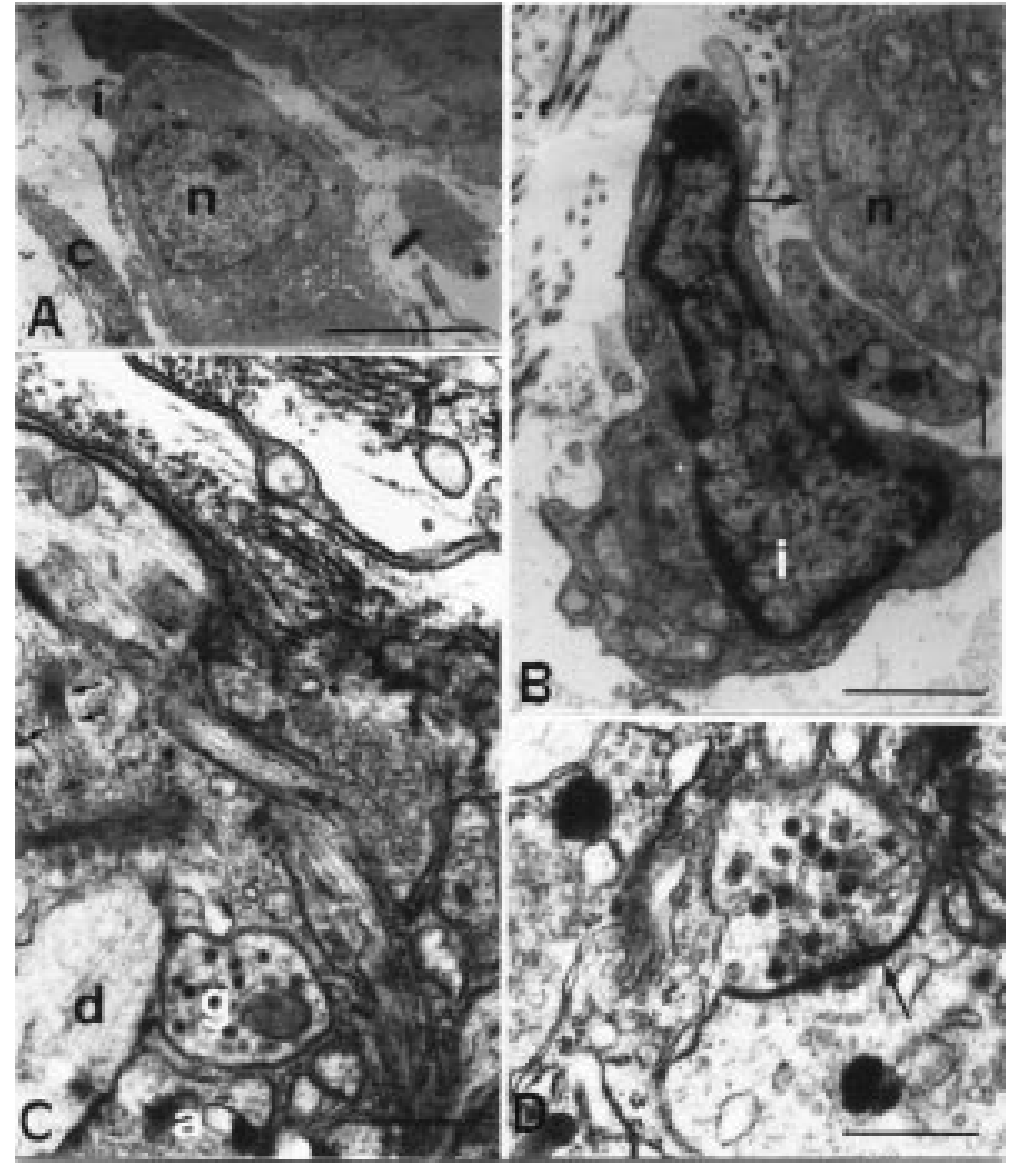


The localization of AChE-activity is reasonable since $\mathrm{AChE}$ has been described as a primary excitatory transmitter to muscle, to intestinal epithelium, to parietal cells, to some gut endocrine cells and to neuro-neuronal synapes (18). Although the presence of this compound suggests the cholinergic nature of these neurons, they need not necessarily be cholinergic since adrenergic and noncholinergic neurons might display AChE positivity (19-21). In fact, noncholinergic functions of AChE have been demonstrated in the brain and AChE was present in DOPAminergic nigrostrial neurons (22). Furthermore, neuronal AChE activity and immunoreactive peptide varicosities were observed simultaneously in the myenteric plexus (23).

The density of nerve cells in the submucous plexus of the mouse has been determined only in the small intestine, with 11,300 neurons $/ \mathrm{cm}^{2}$ for the duodenum, 10,285 neurons $/ \mathrm{cm}^{2}$ for the jejunum and 6,300 neurons/ $\mathrm{cm}^{2}$ for the ileum (16). All the values obtained for the small intestine of Calomys callosus are lower than those observed for the mouse (16).

In general, the neuronal density is higher in smaller than in large animal species. The highest density occurs in the small intestine of the mouse, with more than 10,000 submucosal neurons per $\mathrm{cm}^{2}$ of serosal surface, and the lowest density occurs in the small intestine of the guinea-pig $(16,24)$, with Calomys callosus having intermediate values.

The data for Calomys callosus indicate that the ganglion and nerve cell density in the submucous plexus in the duodenum is higher than that in the other portions of the gut and there is a caudal descending gradient in nerve cell density of the submucous plexus along the small intestine. These results are similar to those obtained for the small intestine of mice (16) and for the cat gastrointestinal tract (25).

The differences in density of nerve cells in the submucous plexus among organs may be correlated with differences in mucosal function (26). The relatively dense submucous plexus of the small intestine is probably related to the capacity of the intestinal mucosa for peptide secretion as well as to absorptive function. Since the major target of the submucosal ganglia is the mucosa, it is possible that the number of submucosal neurons is related to the decrease in the volume of the mucosa (16).

The size of submucosal neurons has not been investigated in mice. In Calomys callosus, the submucosal perikarya display a wide range of sizes and this is true for all five portions of the gut. A population of small neurons is present in all five portions of its digestive tract, whereas the largest neurons are observed in the large intestine rather than in the small intestine.

The present results on the fine structure of the submucous plexus of the intestine of Calomys callosus show that the general organization of the plexus is not dissimilar from that described for other species.

Fibroblast-like cells were found in at least six different locations. Little is known about the factors controlling intestinal fibroblast shape and size. The criteria for distinguishing these cell types from one another are at present rather vague, and cells of intermediate appearance can be found (16). Rather than representing a fixed set of static cell types, it may be that all intestinal fibroblasts have a common origin, and that their dynamic structure and function depend on their local microenvironment (16).

The role played by nerve cells and glial cells in secreting and maintaining the basal lamina surrounding the ganglia and connecting nerve strands is unknown. Recent immunohistochemical evidence suggests that the basal lamina of enteric nerves processes combinations of laminin, type-IV collagen, nidogen, heparin sulfate proteoglycan, and fibronectin similar to those of sensory and sympathetic ganglia, but different from those of smooth muscle cells 
(27). We were able to observe the basal lamina directly around the submucosal ganglia and its appearance was similar to that described in other tissues (28).

The present results show that the submucous plexus of the digestive tract of the wild rodent Calomys callosus contains a large number of neurons of different sizes. The structure of the ganglia is similar to that of other rodent species. The ganglia are covered by a thin layer of collagen fibers and blood vessels are not found within them.

\section{References}

Liberti EA (1997). Morphometry and acetylcholinesterase activity of the myenteric plexus of the wild mouse Calomys callosus. Brazilian Journal of Medical and Biological Research, 30: 627-632.

11. De-Souza RR, Maifrino LBM, Borges N, Souza HM \& Liberti EA (1993). Enteric neuron number and sizes in Auerbach's plexus in the colon of $T$. cruzi chronically infected mice and Calomys callosus. Memórias do Instituto Oswaldo Cruz, 88 (Suppl): 109 (Abstract).

12. Gabella G (1969). Detection of nerve cells by a histochemical technique. Experientia, 25: 218-219

13. Zar JH (1984). Biostatistical Analysis. 2nd edn. Prentice-Hall, New Jersey.

14. Karnovsky MJ \& Roots LA (1964). A "direct-colouring" thiocoline method for cholinesterases. Journal of Histochemistry and Cytochemistry, 12: 19-21.

15. Evan AP, Dail WG, Dammrose D \& Palmer C (1976). Scanning electron microscopy of cell surfaces following removal of extracellular material. Anatomical Record, 185: 433-446.

16. Gabella G (1987). Structure of muscles and nerves of the gastrointestinal tract. In: Johnson LR (Editor), Physiology of the Gastrointestinal Tract. Raven Press, New York.

17. Bor-Seng-Shu E, Chadi G, Bor-Jiun-Shu F, Ferraz-de-Carvalho CA \& de-Souza RR (1994). Myenteric neurons of the mouse small intestine. Morphometry and acetylcholinesterase activity. Brazilian Journal of Medical and Biological Research, 27: 101-108.

18. Furness JB, Bornstein JC, Murphy R \& Pompolo $S$ (1992). Roles of peptides in transmission in the enteric nervous system. Trends in Neurosciences, 15: 66-71.

19. Eranko O, Rachardt L, Eranko L \& Cunninghan A (1970). Light and electron microscopic histochemical observations on cholinesterase containing sympathetic nerve fibers in the pineal body of the rat. Histochemical Journal, 2: 479-489.

20. Levey Al, Wainer BH, Mufson EJ \&
Mesulan MM (1983). Co-localization of acetylcholinesterase and choline acetyltransferase in the rat cerebrum. Neuroscience, 9: 9-22.

21. Seelig LL, Doody P, Brainards L, Gidda JS \& Goyal RK (1984). Acetylcholinesterase and choline acetyltransferase staining of neurons in opossum oesophagus. Anatomical Record, 209: 130-135.

22. Greenfield SA (1985). Acetylcholinesterase release from dendrites. Neuroscience Letters, 22 (Suppl): S3 (Abstract).

23. Anglade $P$, Michel C, Ozaki T, Tsuji $S$, Vignon X \& Yanaihara N (1988). Simultaneous localization of acetylcholinesterase activity and met-enkephalin, vasoactive intestinal peptide and substance $\mathrm{P}$ immunoreactivity in the rat myenteric plexus. Histochemical Journal, 20: 464-467.

24. Liberti EA, Queiroz LM, Pompeu E, Perito MAM, Minarelli AM, Moraes JOR \& De Souza RR (1994). A quantitative and comparative study of the ganglionic neurons in the myenteric and submucous plexuses of the small intestine and in the intramural plexus of the gall bladder of the guinea-pig. Revista Brasileira de Ciências Morfológicas, 11: 106-114.

25. Christensen J \& Rick GA (1985). Nerve cell density in submucous plexus throughout the gut of cat and opossum. Gastroenterology, 89: 1064-1069.

26. Christensen J \& Rick GA (1987). Intrinsic nerves in the mammalian colon: confirmation of a plexus at circular muscle-submucosal interface. Journal of Autonomic Nervous System, 21: 223-231.

27. Bannermann PG, Mirsky R, Jessen KR, Timpl R \& Duance VC (1986). Light microscopic immunolocalization of laminin Type IV collagen, nidogen, heparan sulphate proteoglycan and fibronectin in the enteric nervous system of rat and guinea pig. Journal of Neurocytology, 15: 733743.

28. Sawada H (1981). Scanning electron microscopy of guinea-pig taenia coli. Biomedical Research, 2 (Suppl): 153-158. 